\title{
Representaciones sociales en una comunidad educativa de la Zona de Amenaza Alta del volcán Galeras (Colombia)
}

\author{
Nasly Anabel Ojeda-Eraso ${ }^{1,2}$; Natalia Jurado-Romero ${ }^{3}$; Elizabeth Ojeda-Rosero ${ }^{4^{*}}$
}

DOI: http://dx.doi.org/10.18273/revbol.v40n3-2018009 (c) (i)

Forma de citar: Ojeda-Eraso, N.A, Jurado-Romero, N., y Ojeda-Rosero, E. (2018). Representaciones sociales en una comunidad educativa de la Zona de Amenaza Alta del volcán Galeras (Colombia). Boletín de Geología, 40(3), 195-210. DOI: 10.18273/revbol.v40n3-2018009.

\section{RESUMEN}

A partir del presente estudio se logró comprender las representaciones sociales frente al volcán Galeras con relación a su organización y sus funciones. La metodología cualitativa utilizada retomó elementos de la etnografía y el interaccionismo simbólico, a partir de observación participante, entrevistas, cuestionarios y talleres, desarrollados con diversos actores como docentes, padres de familia y estudiantes. Los resultados se validaron con la población en diferentes espacios y momentos del proceso. Se concluyó que las representaciones sociales frente al volcán Galeras dan cuenta de una perspectiva psico-socio-cultural del fenómeno, interpretándose desde la dialéctica entre el saber popular y el conocimiento científico, que cumple funciones de identidad y de orientación entre los miembros de la comunidad.

Palabras clave: Colombia; volcán Galeras; representaciones sociales; comunidad educativa.

\section{Social representations in an educative community located at High Hazard Zone of Galeras volcano (Colombia)}

\begin{abstract}
Based on research processes, it was possible to understand the social representations related to the Galeras volcano in relation to its organization and its functions. The qualitative methodology returned items of ethnography and symbolic interactionism, from participant observation, interviews, questionnaires and workshops, developed with several actors such as teachers, parents and students, while the results were validated through their continuous participation in the research. It was concluded that social representations related to the Galeras volcano show a psycho-socio-cultural perspective of the phenomenon, understanding from the dialectic between the popular and the scientific knowledge, which have important functions of identity and orientation among the members of the community.
\end{abstract}

Keywords: Colombia; Galeras volcano; social representations; educational community.

\footnotetext{
${ }^{1}$ Universidad de Nariño, Pasto, Colombia. nanabel124@hotmail.com

${ }^{2}$ Corporación Universitaria Minuto de Dios, Pasto, Colombia.

${ }^{3}$ Instituto Colombiano de Bienestar Familiar, Centro Zona Tuquerres, Nariño, Colombia. naatii870517@gmail.com

${ }^{4}$ Grupo de Investigación CONEPSI, Departamento de Psicología, Universidad de Nariño, Pasto, Colombia. (*) deliza75@yahoo.com
} 


\section{INTRODUCCIÓN}

Se desarrolló un estudio desde la psicología social comunitaria, en relación con la presencia de un volcán activo y los sentimientos, pensamientos y significados que se configuran en las personas que habitan en sus faldas; para ello se retomaron elementos metodológicos de la etnografía que dan cuenta del fenómeno desde la perspectiva de la misma gente (Restrepo, 2016). A partir de esta aproximación se lograron entender las prácticas y los significados atribuidos por la comunidad de Genoy al volcán Galeras, vinculando el interaccionismo simbólico como fundamento de la construcción de significados sociales a partir de la interacción entre las personas.

El volcán Galeras se ubica aproximadamente a $9 \mathrm{~km}$ al occidente de la ciudad de San Juan de Pasto; con las siguientes coordenadas: latitud $1^{\circ} 13{ }^{\prime} 43,8^{\prime \prime}$ norte y longitud $77^{\circ} 21^{\prime} 33,0^{\prime \prime}$ oeste y con una altura de 4276 msnm (FIGURA 1); actualmente se encuentra en nivel de alerta III, (amarilla) que se traduce en una posible emergencia por actividad volcánica moderada (Servicio
Geológico Colombiano, 2018), siendo considerado actualmente uno de los volcanes más activos de Colombia y generando constante peligro para las poblaciones que viven en sus cercanías. No obstante, al relacionarlo con los aspectos socio-culturales del contexto en el cual se encuentra ubicado y de acuerdo al estudio desarrollado por David (1996) "influye en quienes se encuentran ubicados en sus alrededores y como se hace evidente, al establecer redes y estructuras que tratan de explicar lo visto y sentido cuando ocurre la reactivación del volcán Galeras" (p. 1).

A lo largo de los años las comunidades que se han asentado en las laderas del volcán han desarrollado una serie de representaciones para explicar los fenómenos de los cuales han sido testigos, encontrando diversidad de expresiones que son un legado cultural para el contexto regional; asimismo, el volcán Galeras se ha catalogado como un punto de referencia para contextualizar la ciudad de San Juan de Pasto, siendo de gran relevancia cuando se habla de la cultura nariñense, de esta manera el fenómeno natural también se ha consolidado como un fenómeno socio-cultural.

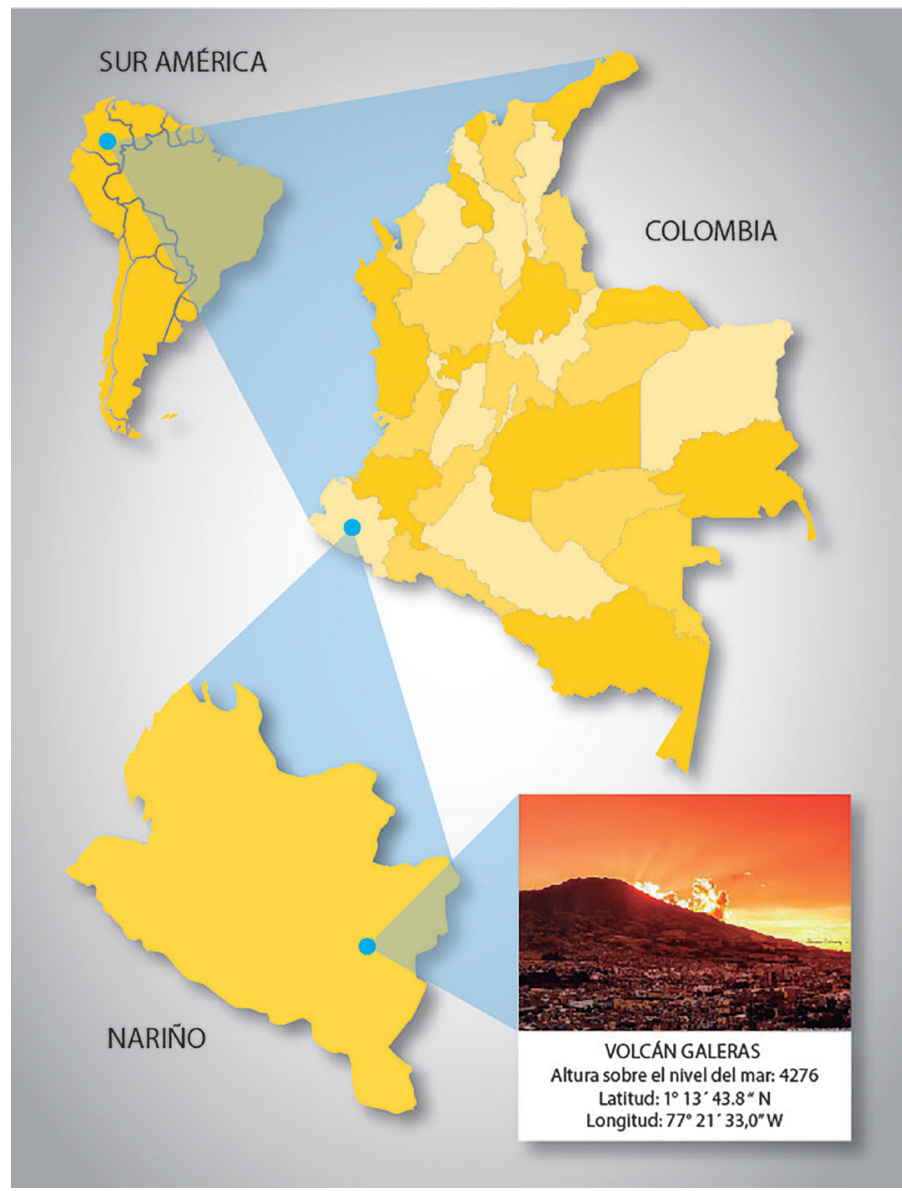

FIGURA 1. Localización del volcán Galeras 
En este orden de ideas, esta investigación se realizó en una comunidad educativa del Corregimiento de Genoy (municipio de Pasto), contexto enmarcado por un amplio bagaje cultural, social e histórico, que se ha inscrito en la memoria de sus habitantes, destacando eventos como las batallas en el tiempo de la conquista, las raíces indígenas (antiguos resguardos), la convivencia con un fenómeno natural como es el volcán Galeras, entre otros; tal convivencia ha suscitado diferentes formas de pensar, sentir y actuar, enmarcadas en lo individual y lo colectivo, que se traduce en saber popular. Este saber se constituye en la base fundamental de una cultura al otorgarle identidad y sentido social, articulándose como una construcción conjunta de la realidad; entendiéndose la cultura, según Banchs et al. (2007) “...como una construcción social, es decir, como patrones estructurados de significados sucesivamente legitimados por las diversas formas de relaciones y prácticas sociales" (p. 64).

En relación a lo anterior y desde la importancia que suscita la cultura para cualquier contexto, surge el estudio del saber popular a través de metodologías etnográficas que buscan acceder a éste y que sea comprensible desde las ciencias humanas y sociales; en los últimos años se ha destacado el estudio de las representaciones sociales, planteado por Serge Moscovici en 1961 y retomado por teóricos como Abric (2001) quien define éstas como un conjunto de informaciones, creencias, opiniones y actitudes relativas a un objeto o situación y compartidas por un grupo de personas.

En esta investigación, la teoría de las representaciones sociales se asumió como un marco pertinente para comprender las diferentes percepciones, creencias, sentimientos y demás elaboraciones presentes en la comunidad educativa del Corregimiento de Genoy, lo que facilitó el acercamiento a la comunidad y su realidad, buscando la comprensión de las relaciones que la comunidad establece con el volcán Galeras, además de promover el respeto por su identidad cultural a través del reconocimiento de los saberes populares que posee, sus tradiciones, idiosincrasia, la relación hombre - naturaleza y que deben ser reconocidas por todas aquellas personas que deseen ahondar o intervenir sobre los habitantes del Corregimiento de Genoy y demás asentamientos humanos en las faldas del volcán Galeras. Cabe mencionar que esta comunidad educativa se conforma con población indígena y campesina, en el caso de la indígena perteneciente a la Parcialidad Indígena Quillasinga de Jenoy.
Junto a la población participante se logró consolidar un proceso dinámico para la comprensión de las representaciones sociales frente al volcán Galeras, enfocándose en reconocer la estructura de dichas representaciones, a partir de la identificación de un núcleo central (principio organizador interno y único que permanece en el tiempo) y el sistema periférico (elementos móviles y jerarquizados que se adaptan a los cambios o retos propuestos por el contexto) y su análisis en el contexto inmediato; de igual manera, se profundizó sobre las funciones que cumplen las representaciones sociales en esta comunidad, como función identitaria y función de orientación (Abric, 2001).

Desde los procesos de investigación cualitativa la participación de la comunidad es el eje transversal para la consecución de los objetivos, conceptualizando la participación comunitaria como un proceso humano que se desarrolla de manera organizada, incluyente y libre caracterizado por gran variedad de participantes, actividades y grados de compromiso y que tienen objetivos compartidos, generando transformaciones individuales y comunitarias (Montero, 2004).

De esta forma, este proceso de investigación se constituyó en un camino y espacio de continuas reflexiones dadas en la comunidad educativa del Corregimiento de Genoy y que invita a ser transitado cuando se quiera acceder a ella, promoviendo el respeto que se debe tener por la diversidad de pensamientos y la cultura de los pueblos como parte importante de sus usos, costumbres e idiosincrasia que legitima a los pueblo indígenas, además se logró contextualizar los fundamentos de la psicología comunitaria en la relación hombre-naturaleza a través de la comprensión del mundo simbólico-cultural y las prácticas manifiestas en las dinámicas sociales y que se expresan en un lenguaje accesible para todos.

\section{METODOLOGÍA}

Se realizó una investigación cualitativa, que según Martínez (2006) "trata de identificar la naturaleza profunda de las realidades, su estructura dinámica, aquella que da razón plena de su comportamiento y manifestaciones" (p. 6), permitiendo un acercamiento complejo y relacional de la situación, con algunas características relevantes para este proceso como el interés por abordar la realidad de forma holística, una comprensión integral que incluye factores políticos, sociales, personales, culturales, históricos, 
biológicos, entre otros; además de promover la crítica y la participación de los actores sociales y donde los resultados se validaron en el consenso, el juicio y la reflexión que se generó.

Dentro de la investigación cualitativa se hizo pertinente un abordaje a partir del enfoque histórico hermenéutico como una actitud comprensiva de la vida humana, parafraseando a López (2001) este enfoque busca ir más allá de lo manifiesto, de lo que se presenta a los sentidos, implica establecer relaciones cruzadas entre fenómenos, sentires, experiencias y generara una percepción integral del evento estudiado, respetando su naturaleza y diversidad; por lo tanto se realizó una comprensión sobre las representaciones sociales que la comunidad educativa constantemente construye frente al volcán Galeras.

Se retomaron elementos metodológicos desde la etnografía, entendiéndose como un proceso de adquisición de conocimiento en una determinada realidad socio-cultural y un producto de la interpretación del conocimiento adquirido por parte del investigador (Comas, 2010); de la misma manera se incluyó el interaccionismo simbólico que Rizo (2004) define como la interpretación por parte de los actores de los símbolos nacidos de sus actividades cotidianas, incluyendo tres premisas básicas: a) las personas actúan sobre la base del significado que atribuyen a los objetos y situaciones que los rodean, b) la significación de estas cosas surge, de la interacción social que un individuo tiene con los demás actores y c) estas significaciones se utilizan como un proceso de interpretación efectuado por la persona en su relación con las cosas que encuentra, y se modifican a través de dicho proceso.

Técnicas: para la recolección de la información se utilizó la observación participante, las entrevistas, los cuestionarios y los talleres. En relación a la estructura de las representaciones sociales, se usaron la asociación libre de palabras y la jerarquización de ítems. La observación participante es la base de la investigación etnográfica, centrándose en la experiencia directa del investigador sobre la realidad y los actores sociales a partir del trabajo de campo y la posterior sistematización de información (Restrepo, 2016).

Dicha técnica se recopiló en el diario de campo, brindando un registro permanente de los procesos dados entre comunidad e investigadoras, lo que permitió anclar los diferentes hallazgos a las situaciones contextuales que rodearon la investigación, consignando elementos relevantes con respecto a las dinámicas de la comunidad. Por otra parte se utilizó la entrevista en profundad como un acto no natural de comunicación susceptible de aprendizaje (Olaz, 2012), teniendo en cuenta características de interacción como disponibilidad, entonación, diálogo fluido y crítico de la situación o temática planteada. También se aplicó la técnica de cuestionario, descrito como un conjunto de preguntas organizadas, claras y con una intencionalidad definida que puede contener preguntas cerradas o abiertas (Araya, 2002); para los propósitos de esta investigación se utilizaron preguntas abiertas ya que éstas no delimitan de antemano las alternativas de respuesta, donde el conocimiento y experiencia del informante es fundamental para complementar y respaldar los hallazgos de las entrevistas. Para la validación del cuestionario se hizo necesaria la participación de la comunidad educativa, a partir de los aportes de personas representativas de la institución educativa como docentes y estudiantes y sus conocimientos en el área y en la región, quienes incentivaron la adecuación de algunos términos del cuestionario para que fuese más comprensible en la población objeto.

En concordancia con los intereses de la presente investigación también se realizaron talleres, entendidos como actividades de participación concertadas para el análisis crítico de la realidad, que posibilitaron comprensiones desde la comunidad como también actitudes personales de empoderamiento y autodeterminación. Coherente con lo anterior Mirebant (2003), considera que un taller es una reunión de trabajo donde se unen los participantes para hacer aprendizajes prácticos según los objetivos que se proponen.

En cuanto a los métodos para el análisis de la información se realizó una aproximación inductiva, es decir un razonamiento ascendente que fluye de lo particular e individual hasta lo general para elaborar una teoría (Abreu, 2014) y posteriormente una triangulación entendiendo esta como el uso de varias estrategias para estudiar el mismo fenómeno, logrando diversos acercamientos e incrementado el panorama del fenómeno humano estudiado (Okuda y Gómez, 2005), por lo tanto se confrontó los hallazgos obtenidos, las teorías existentes, las perspectivas metodológicas desde las cuales se abordó este estudio, complementándose con el análisis de las investigadoras.

Participantes: La investigación se desarrolló con la participación de docentes, estudiantes y padres 
de familia de la Institución Educativa Municipal Francisco de la Villota Corregimiento de Genoy sede bachillerato, por ser la población más numerosa $\mathrm{y}$ donde confluyen diferentes categorías de análisis a partir de ciclo vital, género y rol socio-laboral que desempeñan dentro de la Institución; teniendo claro que la investigación cualitativa está orientada hacia el análisis de casos concretos en su particularidad espacio temporal, y a partir de las expresiones y actividades de las personas en sus contextos locales (Flick, 2012).

El primer grupo poblacional, padres de familia con edades promedio entre 30 y 45 años, habitantes del Corregimiento de Genoy, dedicados principalmente a actividades del hogar, trabajo en la agricultura, y el turismo. Los espacios de acercamiento se establecieron a partir de reuniones convocadas por la institución educativa, encuentros informales dentro y fuera de la institución y favorecidos por el interés de la comunidad en hacer parte de la investigación. En cuanto a la población estudiantil, se vincularon desde el grado sexto a once a través de las diferentes actividades planteadas, a partir de las cuales se identificó que la mayoría de los estudiantes son habitantes del corregimiento, sus edades oscilan entre los 11 y 19 años, con prevalencia del género masculino. El personal docente presente en la institución educativa de la sede básica secundaria se encuentra conformado por 18 personas, la mayoría de ellos no son habitantes del corregimiento, pero con un trabajo de varios años en la institución, lo que les ha permitido tener un mayor acercamiento a los modos de vida de la comunidad, adhesión a usos y costumbres y a la vez tener un panorama amplio de la relación diádica del Corregimiento de Genoy con el volcán Galeras.

\section{RESULTADOS}

A partir de la observación participante se pudo entender dinámicas de interacción propias del contexto educativo, donde sobresalen comportamientos propios de la edad escolar como inicio de relaciones sentimentales, conflictos entre grupos de pares, rebeldía, no aceptación de normas, conciliación permanente ante las dificultades, comportamientos asertivos, apoyo académico, trabajo en grupo; situaciones mediadas por el respeto, la tolerancia, el compañerismo, la solidaridad y el sentido de pertenencia a la institución, dinámicas que favorecen la poca deserción escolar. Además se observó prácticas institucionales encaminadas a fortalecer elementos culturales propios de la región, como mingas del pensamiento (es una propuesta común resultante de los lazos de unidad entre los pueblos o personas para comprender y hallar soluciones a diferentes situaciones), fomento de la creatividad, compromiso hacia eventos como las fiestas patronales del corregimiento, análisis crítico de la realidad y facilitar espacios para el desarrollo de actividades alternas tales como prácticas universitarias, escuela de música, juegos inter-cursos e inter-colegiados y grupo de danzas, todo ello como búsqueda constante del bienestar integral y la participación tanto en lo individual como en lo colectivo.

En relación con la investigación se encontró adaptación de algunas prácticas institucionales hacia el conocimiento científico sobre el volcán Galeras, con mensajes gráficos y textuales referentes al tema, características particulares del contexto como: brigada estudiantil, horario especial cuando hay reactivación del volcán Galeras, implementos de emergencias (botiquines, extintores), material de apoyo, señalización de evacuación en caso de presentarse alguna emergencia; igualmente se hace evidente una divergencia entre el conocimiento aprendido y los actuares, puesto que son escasas las acciones y actitudes hacia la prevención.

Cabe resaltar que durante el proceso de investigación se generó buena disposición por parte de la institución tanto en docentes como en estudiantes para el desarrollo de las actividades planeadas; también el interés de algunos en aprovechar al máximo este proceso como una oportunidad de fortalecimiento personal e institucional y generar espacios de discusión frente a la cantidad de información continuamente recibida.

Con base en las entrevistas, las cuales se desarrollaron a manera de conversaciones espontáneas entre entrevistador y entrevistados en la comunidad educativa se vislumbró variedad de sentimientos y prácticas que ellos han construido como habitantes históricos de las faldas del volcán, reflejados en tradición oral, ritos, costumbres, discursos y prácticas cotidianas, que de alguna manera motiva el continuar viviendo en esta zona a pesar de que otros la denominen como zona de amenaza. A continuación se presentan narrativas que reflejan estos sentimientos y vivencias de la comunidad en torno al volcán Galeras:

Mito: (la estudiante Martínez, D., comunicación verbal) relata que antes vivían por el volcán unos diablos que jugaban chaza, un día un señor fue a ver si era verdad y entonces los diablos lo invitaron a jugar y él dijo que si y entonces como el señor ganó le dieron carbón y él dijo para qué carbón, entonces dejó 
regando un poco y sólo llevó un poco y al amanecer el miró el carbón y era oro. Cuando fue a ver el carbón que había dejado ya no lo encontró, como tampoco a los diablos que jugaban chaza.

Rito: (la señora Guevara, S. comunicación verbal) describe "que cuando erupciona y llueve mucho relampaguea en el volcán, se hacen rezos con la pasada de la Virgen del Rosario en cada casa, colocando altares, lo único que hace la comunidad de Genoy es rezar y pedir a Dios por su bienestar”.

Como se evidencia en los relatos, se destaca la connotación simbólica que tiene el volcán entre las personas, además de la pertenencia que se ha establecido con éste a lo largo del tiempo, donde el temor, el poder y el respeto se encuentran estrechamente ligados, "los rituales aparecen como cultura encarnada, interiorizada, cuya expresión es el dominio del gesto, de la manifestación de las emociones y la capacidad para presentar actuaciones convincentes ante otros" (Rizo, 2004, p. 8).

De la misma forma, los diálogos evidencian que la temática relacionada con el volcán Galeras ha perdido interés, quizá por las reiteradas intervenciones en el tema, con respuestas como "no sé", "mejor pregúntele a...", "no tengo tiempo"; por otra parte, también es notable la dificultad que tienen algunos estudiantes para expresar opiniones propias, limitándose en muchas ocasiones a reiterar ideas dichas por otros (clase, charlas informales, talleres).

En relación a la población docente, asumieron la presente investigación como una oportunidad para debatir, dar a conocer y enriquecer sus posiciones y actitudes frente al volcán Galeras. Se reconoce en ellos su posicionamiento frente al conocimiento científico y la importancia de impartirlo en la institución educativa, reconociendo al volcán Galeras como fenómeno natural impredecible y un posible peligro para toda la comunidad habitante de sus faldas; por ende sus acciones como docentes están encaminadas a prevenir situaciones de riesgo para la comunidad; aunque también han adquirido conocimientos, respetan el saber que posee la comunidad de Genoy y promueven actividades que aportan a la preservación de la tradición.

Al aplicar los cuestionarios, se obtuvieron criterios recurrentes de respuesta que presentan al volcán
Galeras desde el aspecto cultural, donde se rescatan características y elaboraciones simbólicas, que se relacionan con lo religioso y son distintivas de la comunidad educativa, por ejemplo "amigo" que se justificó desde la convivencia, donde los ciclos de actividad volcánica que ha tenido Galeras no han representado amenaza sustancial para la comunidad de Genoy, por el contrario a través de los años se ha consolidado un sentido de pertenencia, respeto y protección hacia el fenómeno. Por otra parte, la comunidad educativa lo percibe como fenómeno natural, es decir, una montaña con lava y ceniza, impredecible, asociando el significante de peligro, desde el conocimiento que continuamente reciben los estudiantes desde diferentes instituciones del estado, coherente con la percepción social generalizada de amenaza frente a cualquier fenómeno natural.

En cuanto a sentimientos de la comunidad se distinguen comportamientos y actitudes de miedo, desacuerdo, incertidumbre, resistencia, asimismo la comunidad estudiantil se ubica simbólicamente como preparados ante una situación de emergencia, siendo conscientes de las características básicas que tiene un fenómeno natural como éste, pero prevaleciendo la idea de que el volcán Galeras no es peligroso.

Al tener en cuenta los gráficos realizados por los estudiantes (FIGURA 2), se hace notorio el conocimiento científico que se ha adquirido sobre el volcán Galeras, corroborando el hecho de estar preparados y que han aprendido como actuar frente a la amenaza, incluyendo variedad de elementos como: organización estudiantil ante emergencias, rutas de evacuación, puntos de encuentro y contando con material que les servirá de apoyo ante cualquier situación. También se encontró percepciones de confianza y tranquilidad en la comunidad educativa que se sustenta en la praxis, en el hecho de que las erupciones del volcán Galeras sean esporádicas, de baja intensidad y que hasta el momento no han tenido graves consecuencias para la comunidad.

También se hizo evidente el interés de la comunidad educativa para que la población externa y entes territoriales perciban la relación que ellos han establecido con el volcán Galeras basada en la confianza, el respeto, la protección del territorio y la amistad, lo que conlleva a que el fenómeno se asuma como un amigo, vecino, protector, un lugar donde se podría fomentar el turismo y que se consolida como parte importante de su región. 


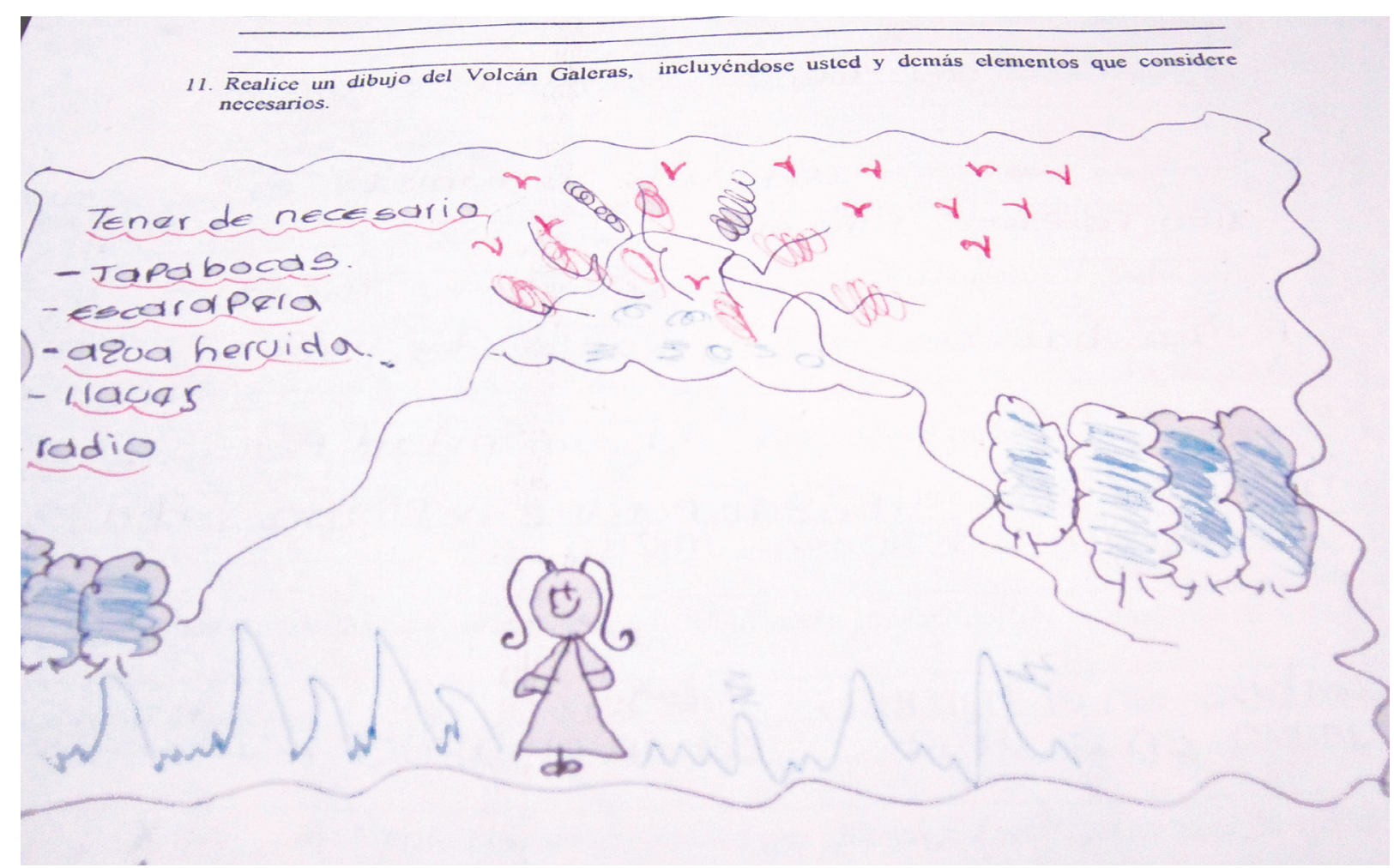

FIGURA 2. Elaboraciones gráficas del volcán Galeras.

En los talleres realizados se contó con la participación principalmente de estudiantes, el objetivo fue profundizar en la lectura de las dinámicas de interacción social, ampliando la visión que tienen sobre sí mismos y sobre su entorno.

Se construyeron algunos significados frente a sus conocimientos y formas de convivencia con el volcán Galeras, los cuales se entienden como representaciones sociales, lo que facilitó la comprensión del saber popular anclado al conocimiento científico presente en ellos al igual que en sus prácticas. Estos talleres fortalecieron la relación comunidad educativa e investigador para lograr interpretaciones más detalladas de la situación estudiada.

También se abordó la problemática en la que actualmente están inmersos, por habitar en una zona considerada de alta y mediana amenaza, según el Mapa de Actualización de Amenaza Volcánica (Servicio Geológico Colombiano, 2015) y las implicaciones personales, sociales, políticas y culturales; al develar las representaciones sociales se evidenció la necesidad de mejorar las estrategias de comunicación dadas entre los miembros de la comunidad y los agentes externos.

\section{Análisis de información en función de la estructura de las representaciones sociales}

Para identificar la organización de las representaciones sociales se tuvo en cuenta el método propuesto por Abric (2001), el cual se caracteriza por retomar datos textuales y estructurarlos de manera tal, que den cuenta de la organización interna de las representaciones sociales (RS).

A partir de la observación participante, talleres, entrevistas, cuestionarios y teniendo en cuenta el análisis de las producciones textuales y verbales de los participantes se encontraron 24 conceptualizaciones recurrentes que representan el volcán Galeras (FIGURA $3)$; la teoría del núcleo central de las representaciones sociales (Abric, 2001) considera relevantes criterios como frecuencia, rango de aparición e importancia del ítem, que se describen a continuación: 


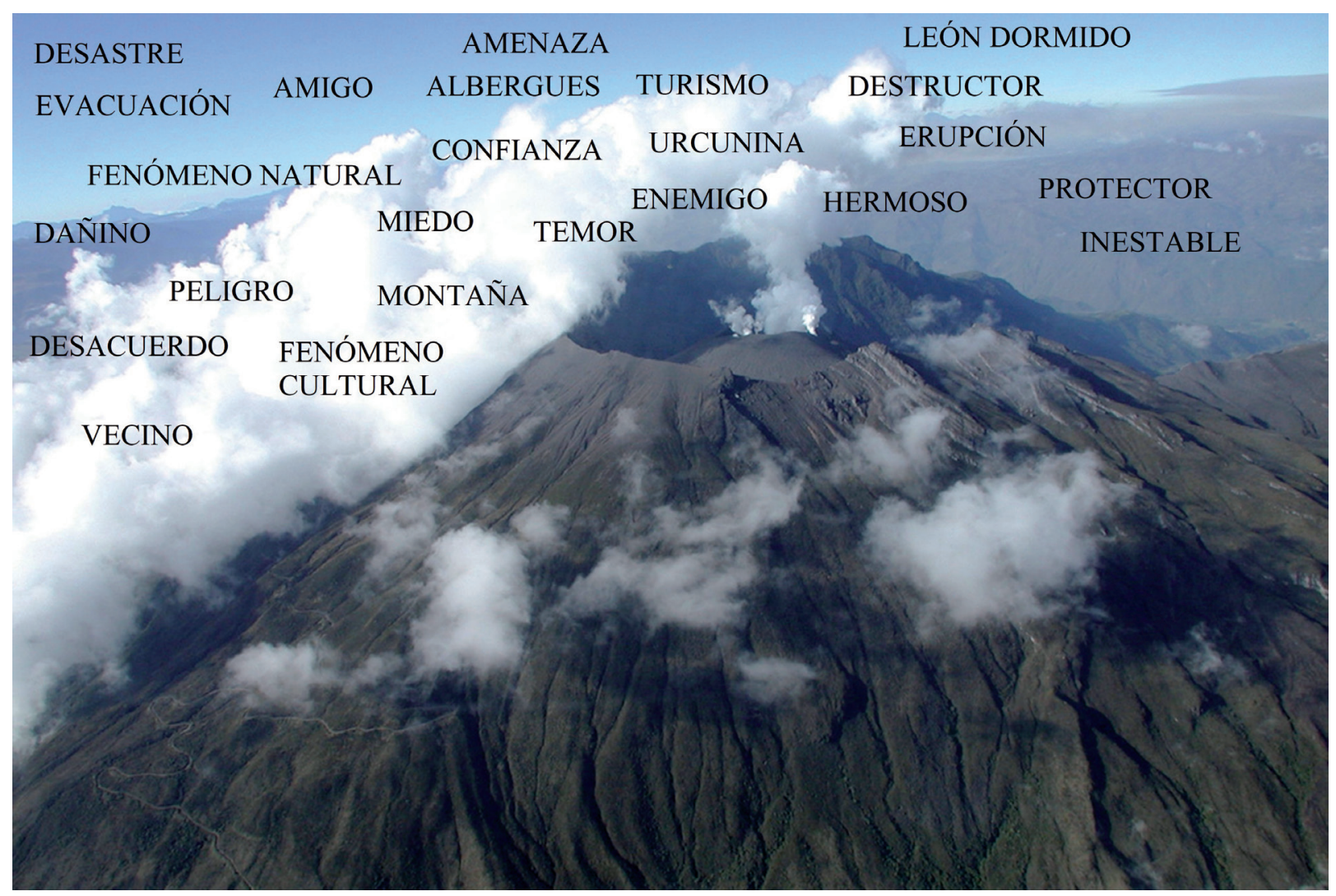

FIGURA 3. Conceptualizaciones recurrentes sobre el volcán Galeras, imagen tomada de Granell (2017).

\section{Asociación libre de palabras}

Teniendo en cuenta los aportes teóricos de Jung (2016) sobre el método de asociación de palabras desarrollado en 1910, se realizan algunas variaciones a la teoría con el propósito de adelantar esta investigación; presentando a los participantes las 24 conceptualizaciones preestablecidas, se sugirió a cada uno la selección de 10 de éstas que consideraran las más significativas al referirse al volcán Galeras, posterior a ello se solicitó que asignaran un valor numérico de 1 a 10, (1 es la más importante y 10 la menos importante) a cada una de las 10 conceptualizaciones.

Teniendo en cuenta las elaboraciones de la población, las conceptualizaciones con mayor frecuencia fueron "amigo y fenómeno natural"; en lo referente al rango de aparición sobresalen conceptualizaciones como "protector y erupción", y finalmente la importancia del ítem que se determinó a partir de la puntuación asignada por los participantes a cada palabra, evidenció que para ellos es importante referirse al volcán Galeras como "amigo, hermoso y fenómeno natural".

De esta manera bajo el método de asociación libre de palabras, se concluyó en acuerdo con la población participante que las conceptualizaciones que más representan su relación con el volcán Galeras son: "amigo, fenómeno natural y protector".

\section{Triadas jerárquicas sucesivas}

Este método planteado por Abric (2001) se basa en la jerarquización de items, para esta investigación se retomaron 24 conceptos recurrentes frente al volcán Galeras y que fueron presentados a la comunidad educativa en forma de fichas. Los participantes debían separar las 24 fichas en partes iguales destacando las más características y las menos características; posteriormente de las doce fichas retenidas por los participantes como las más características se continuaba con la selección hasta llegar a una ficha, que se asumía 
como la más representativa (FIGURA 4). La correlación positiva frecuencia-rango medio, es un indicador de relevancia para captar los elementos centrales de la representación en el grupo estudiado (Abric, 2001).
A partir de esta clasificación se encontró que la comunidad educativa define al volcán Galeras como "fenómeno natural" como se presenta en la TABLA 1.

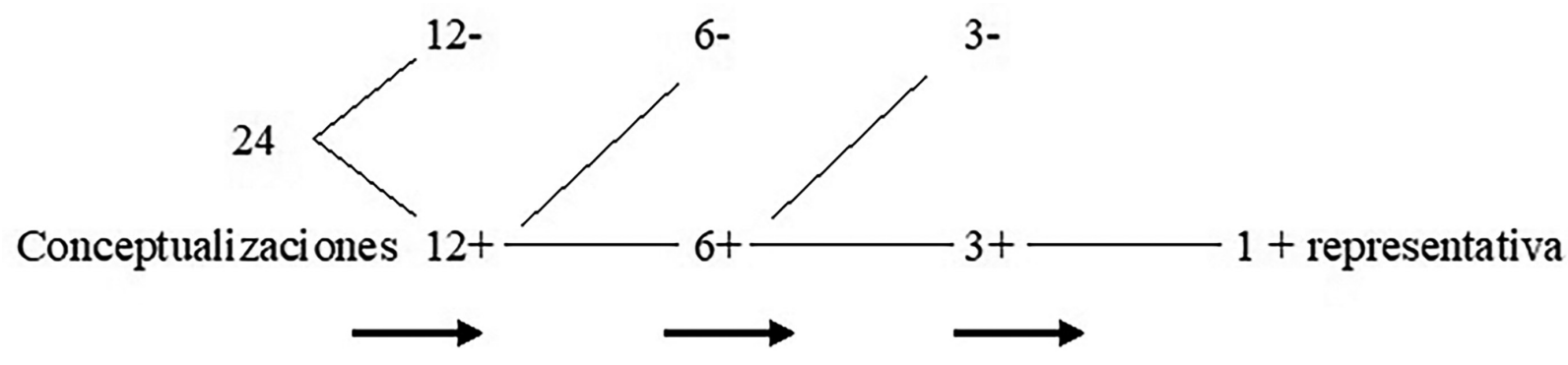

FIGURA 4. Ilustración sobre la fase de jerarquización de ítems realizada por la comunidad educativa sobre el volcán Galeras, partiendo de los aportes teóricos sobre Triadas Jerárquicas Sucesivas (Abric, 2001).

TABLA 1. Comparación de técnicas para la estructuración de las representaciones sociales.

\begin{tabular}{|c|c|c|}
\hline \multicolumn{3}{|r|}{ Análisis comparativo } \\
\hline $\begin{array}{l}\text { Asociación } \\
\text { libre de } \\
\text { palabras }\end{array}$ & $\begin{array}{c}\text { Amigo, } \\
\text { fenómeno } \\
\text { natural y } \\
\text { protector }\end{array}$ & $\begin{array}{l}\text { Desde las asociaciones libres que los participantes realizaron, se concluyó } \\
\text { que es prevalente la apreciación del volcán Galeras desde una perspectiva } \\
\text { cultural, a través de verbalizaciones como "amigo", puesto que para ellos } \\
\text { éste se ha consolidado como un miembro más de su comunidad, con quien } \\
\text { conviven y les resulta difícil concebirse como comunidad desligada del } \\
\text { volcán; así mismo es prevalente la concepción de "fenómeno natural", } \\
\text { entendido como el reconocimiento que la comunidad hace de las } \\
\text { características físicas presentes en el volcán Galeras, como lo inestable e } \\
\text { impredecible de los eventos eruptivos, además se encontró el significante } \\
\text { de "protector" que dentro de la comunidad hace alusión al carácter mágico } \\
\text { que se la otorgado, posicionándolo como un ente que cuida de ellos y al } \\
\text { cual deben respetar como símbolo de autoridad. }\end{array}$ \\
\hline $\begin{array}{l}\text { Triadas } \\
\text { jerárquicas } \\
\text { sucesivas }\end{array}$ & $\begin{array}{c}\text { Fenómeno } \\
\text { natural }\end{array}$ & $\begin{array}{l}\text { La comprensión que la comunidad educativa ha elaborado sobre el volcán } \\
\text { Galeras incluye características físicas que parten de su convivencia con } \\
\text { el fenómeno al ser testigos constantes de sus manifestaciones: emisión } \\
\text { de gases, caída de piroclastos (ceniza, lapilli), sismos volcánicos, sobre } \\
\text { las cuales han creado explicaciones que evidencian una relación directa } \\
\text { entre la comunidad y el fenómeno. Incluyendo en esta concepción los } \\
\text { aprendizajes que ha consolidado la comunidad educativa para afrontar } \\
\text { una posible actividad volcánica, tales como evacuación, organización } \\
\text { social y preparación individual, familiar y comunitaria. }\end{array}$ \\
\hline
\end{tabular}

\section{Estructura de las representaciones sociales}

A partir de la interacción continua con la comunidad educativa con la intención de profundizar en su realidad sociocultural, se logró identificar variedad de expresiones que convergen en las representaciones sociales frente al volcán Galeras, lo anterior en coherencia con los principios etnográficos y de interaccionismo simbólico que se focalizan sobre las dinámicas de interacción presentes en el contexto. De esta manera al tener en cuenta prácticas, sentimientos, cogniciones, creencias y actitudes, se hizo evidente la confluencia de aspectos histórico-culturales al igual que los relacionados con el conocimiento científico sobre el volcán Galeras. 
Núcleo central de las representaciones sociales frente al volcán Galeras

El núcleo central de las representaciones sociales frente al volcán Galeras da cuenta de la "perspectiva psico-socio-cultural" que ha elaborado la comunidad educativa sobre el fenómeno. En lo concerniente a lo "psicológico" refiere a las comprensiones personales y comunitarias que se han construido para relacionarse con el volcán Galeras, mediante procesos sociocognitivos como la adaptación, la resiliencia, el empoderamiento y la auto percepción, que no puede ser entendido sin el contexto, es ahí donde aparece lo "social" como compartido, un significado construido y legitimado entre los miembros de la comunidad, quienes han incluido al fenómeno como un miembro más de su pueblo, que con el transcurso de los años se ha consolidado en su cotidianidad como parte fundamental de sus relaciones entre si y hacia terceros, que también tiene una connotación "cultural" al ubicar al volcán Galeras como centro de sus dinámicas sociales, otorgándole vida y explicación a la actividad volcánica a partir del comportamiento de la comunidad. Esta perspectiva psico-socio-cultural se puede explicar desde la estrecha relación existente entre hombrenaturaleza presente en los pueblos indígenas, como una visión trascendente del hombre, la vida; "los pueblos indígenas han caminado a la par del tiempo y la naturaleza, proyectan su cosmovisión en la que tiempo y espacio están articulados, pero también envían el mensaje de que ellos están en estrecha relación con la naturaleza" Caudillo (2010, p. 7). De tal manera que en la comunidad educativa confluye el saber popular y conocimiento científico, interpretando el volcán como "un fenómeno natural que no implica amenaza", puesto que desde su historia de convivencia hasta el momento no han ocurrido eventos volcánicos de gran magnitud que representen peligro, sin embargo y dado su carácter impredecible la comunidad educativa se siente preparada para afrontar dichos eventos, reconociendo las acciones pertinentes ante este tipo de situaciones (preparación, contingencia, evacuación y mitigación ante el peligro).

\section{Sistema periférico de las representaciones sociales frente al volcán Galeras}

Cultura presente en la comunidad educativa: a partir de elementos culturales como cogniciones y prácticas, que se evidenciaron en el transcurso de la investigación y se enlazan con la importancia que la comunidad educativa da al fenómeno, desde una concepción simbólica, donde se mantienen ritos y tradición oral que combinan historia e imaginación y que están presentes en representaciones como amigo, protector y vecino, en los cuales se enlaza la tradición familiar y lo religioso, además evidente en prácticas como: fiestas patronales, procesiones, misas, altares, ofrendas que se enmarcan en la religión católica así como también en sus conversaciones "el volcán es mi amigo", "nunca nos ha hecho nada", "yo hago lo que mi familia diga, si dice que nos vamos yo me voy y si no, no", "la virgen nos cuida" que permiten identificar coherencia entre prácticas y sentires sobre el volcán Galeras.

Conocimiento científico sobre el volcán Galeras: además de la cultura ancestral presente en la comunidad de Genoy, se hizo notorio el conocimiento científico que actualmente instituciones gubernamentales y privadas han promovido en la institución educativa, con el ánimo de prevenir situaciones de peligro en la comunidad del Corregimiento de Genoy que habita en la zona de amenaza volcánica alta y media (Servicio Geológico Colombiano, 2015). Se hicieron evidentes cogniciones que dan cuenta del conocimiento científico que han adquirido al relacionarse con estas instituciones, tales como evacuación, peligro y prevención, por lo anterior se esperaría acciones a nivel personal, familiar y comunitario tendientes hacia la prevención que en la práctica no son muy evidentes. También se encontró actividades propias de la institución educativa tendientes a promover prácticas de prevención, como: implementos básicos, lugares de evacuación, organización comunitaria, simulacros y brigadas, entre otros. Es así por ejemplo, que cuando hay cambios en la alerta volcánica la institución ha preparado un horario especial, que los estudiantes asumen con relativa normalidad e incluso como una oportunidad de evadir responsabilidades académicas; de esta manera se diría que hay contradicciones entre cogniciones y prácticas, desde la teoría se podrían interpretar como inclusiones o adaptaciones en el sistema de representaciones sociales, para anclarse al contexto inmediato; en la FIGURA 5 se puede encontrar el esquema de las representaciones sociales para la comunidad educativa de Genoy. 


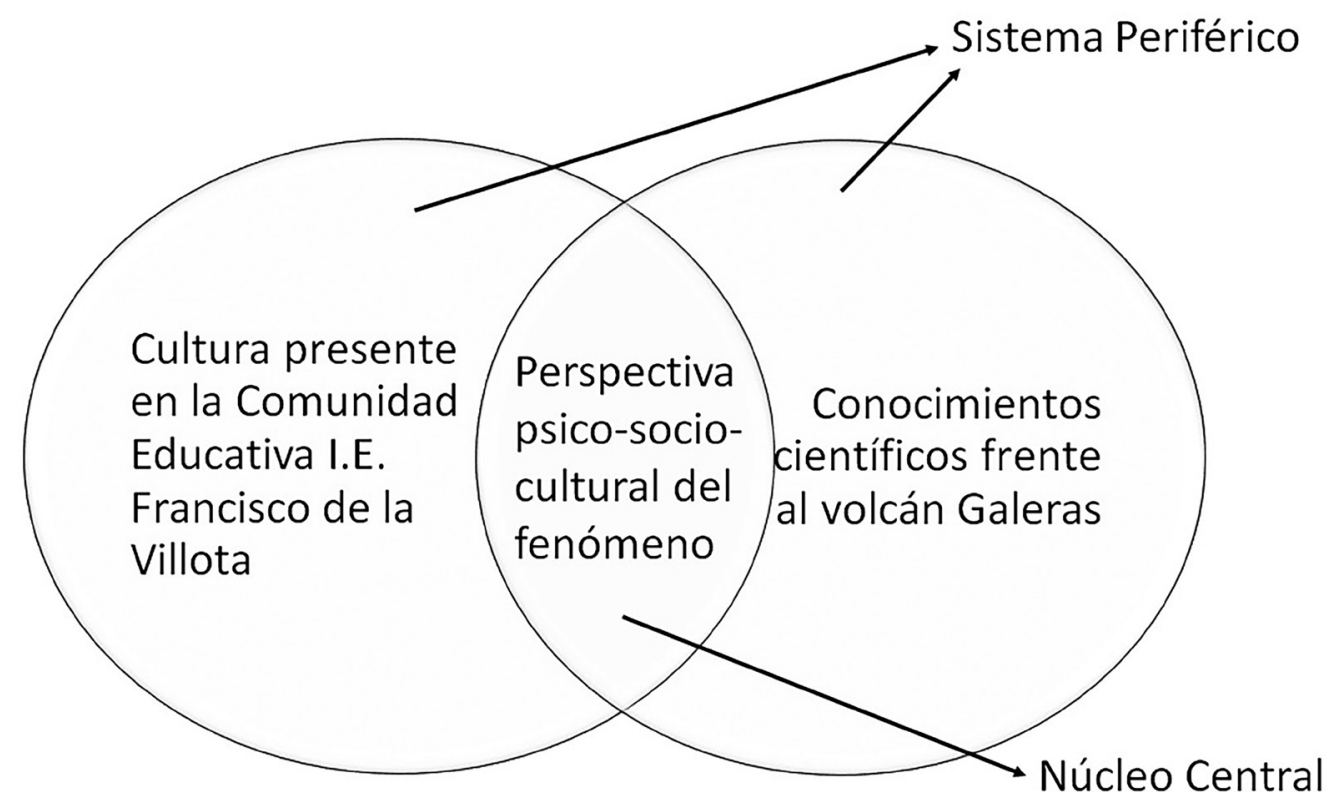

FIGURA 5. Estructura de las representaciones sociales.

\section{Funciones de las representaciones sociales frente al} volcán Galeras

Durante el proceso de investigación se reconoció a través de sus producciones, formas de convivencia, prácticas cotidianas, comunicaciones y modos de interacción dados en la comunidad educativa, que las representaciones sociales frente al volcán Galeras cumplen una función de identidad, entendida ésta como la elaboración de una identificación social y personal gratificante; es decir, compatible con los sistemas de normas y valores social e históricamente determinados, que para el caso de la comunidad educativa de Genoy se encamina a proteger su riqueza histórica y cultural, que también se asocia con el fenómeno y a la vez contribuye a la defensa ante la influencia de la modernidad incluido el conocimiento científico, que para la comunidad educativa implica cambios sustanciales en sus formas de vida. De igual manera es evidente la función de orientación de comportamientos, presente en la tradición cultural, las acciones de respeto hacia el volcán Galeras, así como también los comportamientos de calma y tranquilidad ante la actividad volcánica y la negativa por abandonar las tierras de las cuales son pobladores históricos, que como pueblos indígenas la defensa y protección de su territorio cobra vital importancia como derecho colectivo, logrado a nivel ancestral y constitucional.

Además de lo planteado anteriormente las representaciones sociales que la comunidad educativa tiene frente al volcán Galeras se han consolidado como un significado compartido, modulando las formas de interacción dadas en la comunidad. Por consiguiente de las interacciones cotidianas entre ellos surgen las representaciones sociales frente al fenómeno y estas a su vez consolidan un significado único y compartido por la comunidad educativa que facilita la integración y las dinámicas sociales. Por otra parte, este sistema de representaciones permite una mirada particular de la comunidad hacia su realidad contextual, que podría dar cuenta y explicar las acciones y concepciones que ellos tienen en relación al volcán y que por muchos no son comprendidas. La realidad se recrea de forma permanente y las representaciones se adaptan a los diferentes cambios que se puedan presentar, al igual que los comportamientos de las personas que comparten dichas representaciones, en este caso la intromisión del conocimiento científico, que promueve un posicionamiento desde la prevención y el reconocimiento de las características físicas del volcán que puede llegar a modificar la representación.

\section{Dinámica de las redes sociales presentes en la comunidad educativa}

Se destaca un flujo constante de información y el fortalecimiento del sistema periférico de las representaciones sociales (FIGURA 6). 


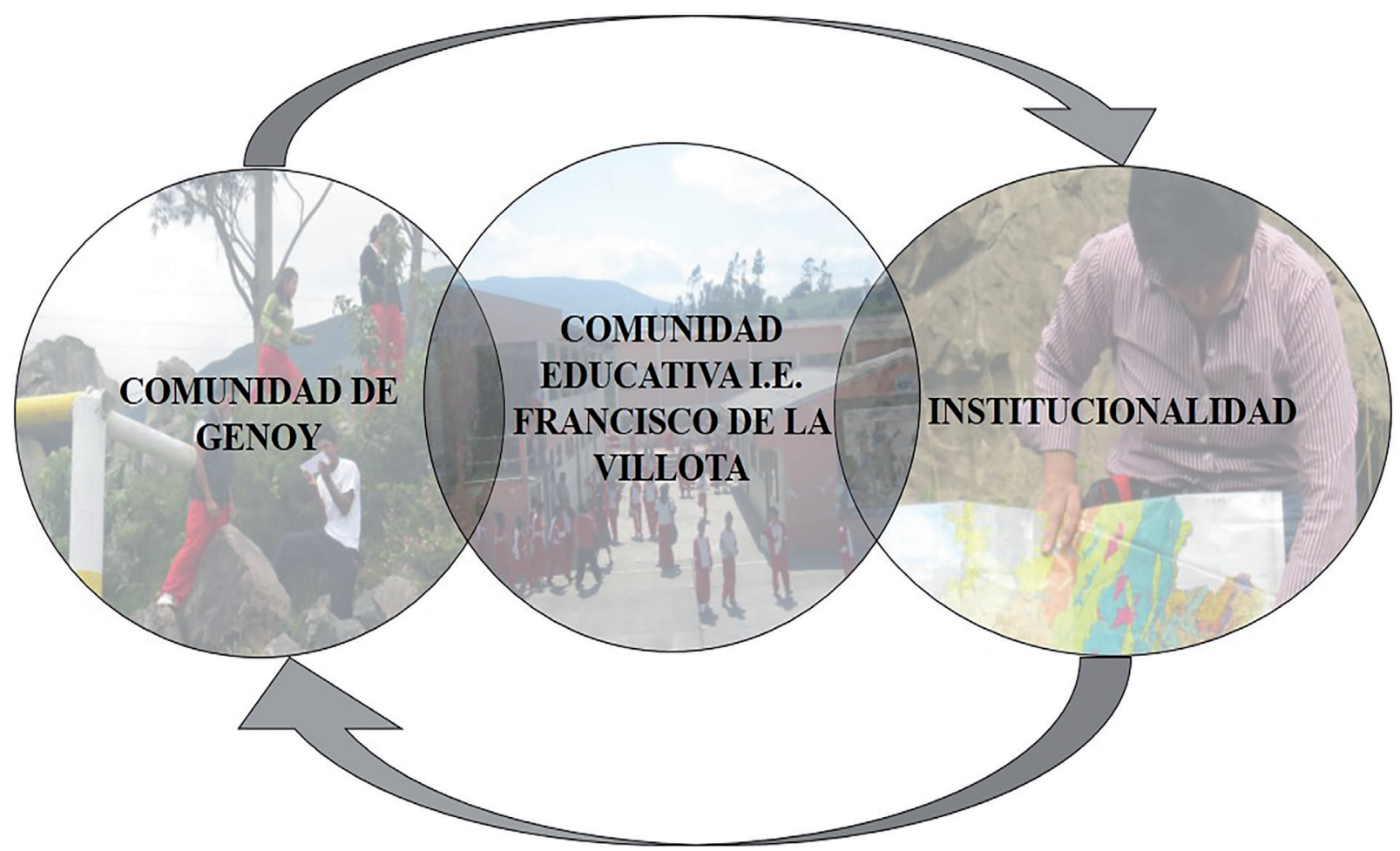

FIGURA 6. Redes de interacción social; en la comunidad educativa confluyen los diferentes grupos sociales presentes (padres de familia, jóvenes, adolescentes y niños) y también las instituciones públicas y privadas (Volcanes de Nariño, Servicio Geológico Colombiano); esta continua interacción favorece el sistema periférico de las representaciones sociales, ampliando las prácticas y conocimiento sobre el volcán Galeras, tanto hacia el saber popular como científico e influyéndose mutuamente.

\section{DISCUSIÓN}

Se realizó una triangulación de información, entre los hallazgos dados en la comunidad, los planteamientos desde la teoría y las elaboraciones de las investigadoras.

En coherencia con las metas de la presente investigación hacia la comprensión de las representaciones sociales, se puede concluir que los hallazgos producto de este proceso con la comunidad educativa consolidaron una significativa comprensión del tema estudiado, en la misma medida que dichos hallazgos dan cuenta de las dinámicas comunitarias que permitieron el surgimiento de las representaciones sociales frente al volcán Galeras, al tomar en cuenta condiciones necesarias para la formación de una representación social. Al respecto, Ruíz (2001) identifica que éstas: a) suponen un cambio importante en la concepción del mundo y del ser humano que es aplicable con el contexto estudiado en la medida que la comunidad crea una concepción particular del fenómeno y de la relación que se ha establecido; este cambio trasciende hacia una cosmovisión de estrecha relación entre hombre y naturaleza que va más allá del ciclo de la vida; b) fenómenos o procesos que modifican las condiciones de vida de una sociedad, produciéndose cambios en las concepciones de los objetos sociales. A partir de la convivencia de la comunidad con el volcán y como testigos de su actividad, surge la necesidad de explicar estos sucesos, por lo tanto ellos crean una serie de concepciones que parten de su saber popular para dar cuenta de lo vivido y sentido, permitiendo así la consolidación de un objeto social identitario. También se retoma los aportes de Moscovici (1979), al proponer que los miembros del grupo presentan un interés particular por ciertos aspectos del objeto social y un desinterés relativo por otros aspectos; de esta manera los miembros de la comunidad asignan mayor interés al carácter socio-cultural y de identidad que tienen con el volcán Galeras y desestiman los aportes dados desde el conocimiento científico; bajo la misma teoría se plantea que los individuos completan lo que no conocen del objeto social con las opiniones dominantes en el grupo (Moscovici, 1979); esto se evidencia en el no reconocer los aportes del conocimiento científico, inclinándose por la concepción compartida de que el fenómeno no es peligroso, partiendo de sus imaginarios colectivos y tradiciones religiosas que complementan y justifican la concepción. 
De la mano con lo anterior, las elaboraciones encontradas refieren a representaciones sociales en tanto el conocimiento implícito es de carácter público, circula libremente en el grupo y se inserta en el discurso cotidiano de las personas (Ruíz, 2001).

\section{Núcleo central de las representaciones sociales frente al volcán Galeras}

Retomando los planteamientos de Abric (2001) el núcleo central es estable, coherente y resistente al cambio y es de cierta manera relativamente independiente en el contexto social y material inmediato. De acuerdo a lo encontrado en la presente investigación se ha establecido desde una connotación cultural que el núcleo central da cuenta de "una perspectiva psico-socio-cultural del fenómeno", sustentada en la historia de la comunidad educativa, ya que no se han presentado transformaciones o cambios significativos que modifiquen el núcleo central y por ende la representación; por el contrario, las situaciones que se han dado en la comunidad lo han fortalecido, siendo coherente con su historia, su cultura y sus relaciones cotidianas con el fenómeno; sumado a lo anterior Abric y Elejabarrieta (Abric, 2001) consideran que el núcleo central se alimenta y relaciona los eventos históricos, sociológicos e ideológicos de la comunidad; se refiere entonces a su memoria colectiva como grupo, teniendo en cuenta que el posicionamiento frente al volcán Galeras parte del conocimiento popular, producto de la convivencia histórica, la riqueza cultural, las formas de interacción y el fortalecimiento de su identidad como grupo, que se entrelaza con las enseñanzas impartidas por la institución educativa e instituciones gubernamentales y privadas, hacia una interpretación del volcán Galeras que va más allá de la concepción física del fenómeno y adquiere un carácter simbólico, propio de la comunidad educativa que guía prácticas y cogniciones. El núcleo central también constituye la base compartida, común de la representación, como una función consensual (Ruíz, 2001) donde la perspectiva psico-socio-cultural abarca las intersubjetividades y refuerza su identidad social, al ser trasmitida en la cotidianidad a través de los diferentes grupos sociales y contextos inmediatos.

En la misma línea de discusión Abric (2001) también propone la característica estructurante del núcleo central, atribuyéndole dos funciones: una función generadora de significados y otra organizadora de las relaciones entre los elementos de la representación; en esta oportunidad se hacen visibles en la capacidad que tiene la perspectiva psico-socio-cultural del fenómeno para incluir y dar cuenta de todas las concepciones y dinámicas que sobre sí confluyen, al integrar los sentires individuales, grupales, prácticas, percepciones, interacciones, formas de expresión y demás, que en el transcurso del tiempo han adquirido un sentido propio para la comunidad educativa, obedeciendo a la cultura y el contexto que se establece como un significado compartido del fenómeno.

De la misma manera Ruíz (2001) propone características del núcleo central como la expresión en formas gramaticales diversas. Para el caso de volcán Galeras, se evidencia en la comunidad en la utilización de términos como Urcunina, León dormido y también significaciones diversas, es decir el fenómeno se percibe como amigo, peligro, protector, entre otras, que varía en relación a las individuales; demostrando el poder asociativo que tienen las representaciones sociales y la capacidad del núcleo central para contener variedad de concepciones.

\section{Sistema periférico de las representaciones sociales frente al volcán Galeras}

En relación a los planteamientos de Abric, el sistema periférico se relaciona con las historias individuales de las personas, influenciadas por el contexto social inmediato, donde los sujetos se desenvuelven; cumpliendo algunas funciones como la concreción que hace referencia al anclaje de la representación en la realidad, integrando elementos de la situación en la que la representación se produce (Abric, 2001), encontrando variedad de cogniciones que parten de las comprensiones individuales y que en conjunto enriquecen constantemente el sistema de representaciones sociales y se hacen visibles en los diálogos cotidianos, en la ritualidad y religiosidad, de igual manera en eventos especiales como festividades patronales o fiestas comunitarias, siendo coherentes con el núcleo central. Los elementos periféricos también aluden a significados más individualizados, en este caso hacia la concepción del volcán Galeras que en la comunidad se debate entre el saber popular y el conocimiento científico (protector o amenaza).

También hace parte del sistema periférico la función de regulación que hace referencia a la adaptación de la representación al contexto inmediato e integrar nuevos elementos, constituyéndose en el aspecto móvil y evolutivo de la representación (Abric, 2001); destacando cambios en el contexto que movilizan cambios a nivel personal y social, siendo las RS constantemente encargadas de integrar a los 
conocimientos y prácticas ya adquiridas, los nuevos aportes desde el entorno de forma coherente y que no implique contradicción; para el caso, el incluir en sus vidas el conocimiento científico sobre el fenómeno, esta adaptación está mediada por las diferencias individuales que se hacen presentes en la comunidad de Genoy en el desacuerdo y ambivalencia que los habitantes experimentan como producto del choque o conjunción entre el saber popular y el conocimiento científico, se debaten entre la defensa de sus territorios ancestrales y la necesidad de abandonar una zona de amenaza volcánica alta.

Igualmente teniendo en cuenta los aportes de Ruíz (2001) sobre el sistema periférico, en tanto resalta que "al depender en gran medida de la historia individual y carecer del carácter compartido, permite la formación de representaciones sociales individualizadas mediante variaciones o apropiaciones individuales de la representación" (p. 39), es decir las personas interpretan constantemente su realidad y elaboran representaciones individuales del fenómeno, por consiguiente, desde esta investigación se asumen en plural, como representaciones sociales frente al volcán Galeras y no como una sola representación social, incapaz de significar el conjunto de perspectivas presentes en la comunidad y que son producto de la relación con el volcán.

De la misma manera se resalta la función de defensa cumplida por el sistema periférico, cuyo propósito es proteger al núcleo central de la amenaza de otros elementos que cuestionan la representación social, incorporando o desechando éstos elementos (Abric, 2001), encontrando así que el conocimiento científico podría colocar en riesgo al núcleo central de las representaciones sociales. Esta función procura incorporar en la perspectiva psico-socio-cultural elementos compartidos con el conocimiento científico sobre el volcán Galeras, tales como la búsqueda del bienestar comunitario, la necesidad de desarrollo regional, la historia de actividad volcánica y la defensa de los territorios, cuyo producto es el enriquecimiento constante de las representaciones sociales y que la comunidad se perciba como preparada ante el peligro que en algún momento podría representar el volcán Galeras. En conclusión el sistema periférico favorece la heterogeneidad del grupo, el desarrollo de las diferencias individuales y permite el crecimiento personal, a la vez que enriquece constantemente las representaciones sociales y las adapta a los cambios en el contexto.

\section{Funciones de las representaciones sociales frente al volcán Galeras}

En la investigación se identificó que las RS cumplen una función identitaria, Abric (2001) la define como "elaboración de una identidad social y personal gratificantes; es decir, compatible con los sistemas de normas social e históricamente determinados, también juega un rol importante en el control social ejercido por el grupo sobre cada uno de sus miembros" (p. 15), reflejado en la comunidad educativa en la medida que constituye cogniciones y prácticas que diferencian positivamente a esta comunidad de otras, diferenciación que surge gracias a la riqueza cultural e histórica presente en la comunidad, puesto que los territorios de Genoy han vivido procesos que marcaron la cultura de la región, como la resistencia dada en el tiempo de la independencia, albergando los ejércitos de Agustín Agualongo quienes efectuaban deliberaciones y planes de ataque. Asimismo el ocultar al padre Francisco de la Villota y protegerle la vida (Mora, 1995), que se relaciona de igual forma con aspectos como el sentido de pertenencia hacia su territorio, lugar donde se han construido tejidos sociales, dinámicas de interacción comunitaria, lazos familiares, lugares simbólicos (iglesia, cementerio, volcán, cueva del padre Villota, la piedra de los sacrificios, las cascadas, el mantel de piedra, entre otros) y que podrían justificar la negativa de la comunidad por abandonar la tierra que durante muchos años ha sido testigo de su desarrollo e historia; el dejarla atrás implicaría abandonar parte de su identidad, que ha logrado mantenerse en el tiempo.

Si bien la principal función que cumplen las representaciones sociales en la comunidad educativa es dar identidad a sus miembros, también se evidencia la función de orientación, que consiste en guiar los comportamientos y las prácticas sociales, prescriben como actuar y en que situaciones (Abric, 2001), evidente en la comunidad educativa del Corregimiento de Genoy a partir de comportamientos sociales como la tradición oral, los rituales ancestrales y religiosos y la confluencia de nuevos conocimientos representados en la cultura de la prevención (evacuación, kit de emergencia, organización social). 


\section{Redes de interacción social}

En las dinámicas de la comunidad educativa son importantes las manifestaciones individuales y grupales, como "un proceso de continua actividad en la que los participantes desarrollan líneas de acción, ante las innumerables situaciones que han de afrontar" (Blumer, 2002, p. 12), de esta manera se crean constantemente significados compartidos que entre otras funciones, dan sentido a la realidad e implícitamente cada uno de los actores sociales tiene un rol en las dinámicas comunitarias; en la misma medida Blumer (2002), plantea que "los participantes están como engranados en un vasto proceso de interacción, en el seno del cual deben hacer que sus acciones en desarrollo se adapten a las ajenas" (p. 12), en este caso la adaptación de las prácticas individuales al sistema predominante en el cual están inmersos y que contribuyen a la adaptación permanente de las representaciones sociales frente al volcán Galeras. También entre las redes sociales presentes en la comunidad educativa, se crean significados particulares para dar cuenta de la relación con el volcán Galeras, concretamente como un código que representa la memoria social de la comunidad y que posibilita la continuidad histórica de la cultura local (Krause, 1999) a través de símbolos que permiten la expresión de sentires y la comunicación transformadora. A manera de ejemplo: la expresión de los sentires frente al volcán Galeras se refleja en los diálogos cotidianos, las canciones, obras de arte, mitos, ritos, poesía y demás, "las personas están en un constante cambio y construcción en su relación dialéctica" (López, 2001, p. 118).

\section{CONCLUSIONES}

La investigación permitió como principal logro un acercamiento a la realidad socio-cultural de la comunidad educativa del Corregimiento de Genoy y el reconocimiento de la riqueza conceptual y diversidad de conocimientos inmersos en la comunidad educativa referentes a la temática Galeras, que se han consolidado en el transcurso del tiempo como también los aportes que la institución educativa brinda a la comunidad.

Se vislumbró en la comunidad educativa el proceso de transición que afronta, desde la confluencia entre la tradición o el saber popular y el conocimiento científico sobre su contexto en relación directa con el volcán Galeras y las implicaciones que este proceso de acoplamiento ha generado para todos los habitantes.
Dentro de la investigación se reconoce que en la comunidad educativa ha sido constantemente abordada la temática Galeras lo que ha generado en ellos sentimientos, actitudes y comportamientos de desinterés; puesto que la mayoría de las intervenciones han sido externas, dadas desde diferentes entes que no facilitan procesos de inclusión y participación comunitaria.

En el estudio de las representaciones sociales en el contexto de Genoy, es de resaltar el sentimiento de identidad que en la comunidad se ha mantenido y consolidado hacia su entorno, marco de referencia que debe tenerse en cuenta y respetarse para cualquier acercamiento.

\section{AGRADECIMIENTOS}

A la comunidad educativa de la Institución Educativa Municipal Francisco de la Villota, Corregimiento de Genoy, Municipio de Pasto, Colombia. A los revisores del artículo por los aportes realizados.

\section{REFERENCIAS}

Abreu, J. (2014). El método de la investigación. Consultado el 10 de abril 2018. http://www. spentamexico.org/v10-n1/A14.10(1)205-214.pdf.

Abric, J. (2001). Prácticas sociales y representaciones. México D.F., México: Coyoacán.

Araya, S. (2002). Las representaciones sociales: ejes teóricos para su discusión. Consultado el 20 de agosto de 2009. http://www.flacso.or.cr/fileadmin/ documentos/FLACSO/Cuaderno127.pdf.

Banchs, M. Agudo, A., y Astorga, L. (2007). Imaginarios, representaciones y memoria social. Espacios imaginarios y representaciones sociales, aportes desde Latinoamérica. Barcelona, España: Anthropos, 64p.

Blumer, H. (2002). El interaccionismo simbólico: perspectiva y método. Consultado el 12 de Julio de 2010. http://eav.upb.edu.co/banco/files/ interaccionismosimbolico.ppt.

Caudillo, G. (2010). Pueblos indígenas y naturaleza: la alternativa del buen vivir. Contextualizaciones Latinoamericanas, 2.

Comas, D. (2010). Etnografía. Barcelona, España: Editorial UOC. 
David, M. (1996). El imaginario en las erupciones del Galeras. Tesis de Maestría, Universidad de Nariño, San Juan de Pasto, Colombia.

Flick, U. (2012). Introducción a la Investigación Cualitativa. Madrid, España: Ediciones Morata.

Granell, J. (2017). Volcanian. Registros e información detallada de los volcanes de nuestro mundo y el más allá. Galeras. Consultado el 20 de septiembre 2017. http://volcanian.blogspot.com/2017/01/ galeras.html.

Servicio Geológico Colombiano. (2015). Actualización del Mapa de Amenaza Volcánica del Volcán Galeras. Consultado el 12 de abril de 2018. https:// www2.sgc.gov.co/sgc/volcanes/VolcanGaleras/ Paginas/Mapa-de-amenaza.aspx

Servicio Geológico Colombiano. (2018). Generalidades Volcán Galeras. Consultado el 10 de enero de 2018. https://www2.sgc.gov.co/sgc/ volcanes/VolcanGaleras/Paginas/generalidadesvolcan-galeras.aspx.

Jung, C. (2016). Obra completa de Carl Gustav Jung. Volumen 2: Investigaciones experimentales. Estudios acerca de la asociación de palabras. Traducción Carlos Martín Ramírez. Madrid: Trotta.

Krause, M. (1999). Representaciones Sociales y Psicología Comunitaria. Consultado el 10 de enero de 2018. http://www.psykhe.cl/index.php/ psykhe/article/download/392/372.

López, H. (2001). Investigación Cualitativa $y$ Participativa. Un Enfoque HistóricoHermenéutico y Crítico-Social en Psicología y Educación Ambiental. 118p. Consultado el 13 de Abril de 2010. http://pub.edu.co/invescua_ documento/pdf.

Martínez, M. (2006). La investigación cualitativa (Sintesis conceptual). Consultado el 5 de Junio de 2010. http://sisbib.unmsm.edu.pe/bvrevistas/ investigacion_psicologia/v09_n1/pdf/a09v9n1.pdf.

Mirebant, G. (2003). El concepto de taller. Consultado el 17 de mayo de 2010. http://acreditación. unillanos.edu.co/contenidos/dis_ambientes_ metodos_pedagogico.
Montero, M. (2004). La participación y el compromiso en el trabajo comunitario. En: Introducción a la psicología comunitaria. Desarrollo, conceptos y procesos (pp. 108-122). Buenos Aires: Paidós.

Mora, C. (1995). Estudio socio-económico de Genoy. Instituto Agrícola "Francisco de la Villota" Corregimiento de Jenoy. Tesis. Universidad del Cauca, Popayán, Colombia.

Moscovici, S. (1979). Psicología Social II. Barcelona, España: Huemul S.A.

Okuda, M., y Gómez, C. (2005). Métodos de investigación cualitativa: triangulación. Consultado el 10 de mayo de 2018. http://www. redalyc.org/articulo.oa?id=80628403009.

Olaz, A. (2012). La entrevista en profundidad, justificación metodológica y guía de actuación práctica. España: Septem Ediciones.

Restrepo, E. (2016). Etnografía: alcances, técnicas y éticas. Bogotá, Colombia: Envión Editores.

Rizo, M. (2004). El interaccionismo simbólico y la Escuela de Palo Alto. Hacia un concepto de comunicación. Consultado el 13 de Julio de 2010. http://www.portalcomunicacion.com/esp/pdf/ aab_lec/17.pdf.

Ruíz, J. (2001). Representaciones sociales: teoría y métodos de investigación; Avances en medición $y$ evaluación en psicología y educación: cinco lecturas selectas. Medellín, Colombia: Universidad de los Andes.

\begin{tabular}{c}
\hline \hline Nasly Anabel Ojeda-Eraso \\
ORCID: 0000-0002-3116-7749 \\
Natalia Jurado-Romero \\
ORCID: 0000-0001-9180-153X \\
Elizabeth Ojeda-Rosero \\
ORCID: 0000-0003-1862-6354 \\
\hline \hline
\end{tabular}

Trabajo recibido: febrero 27 de 2018

Trabajo aceptado: mayo 30 de 2018 